Sharif University of Technology
Scientia Iranica
Transactions E: Industrial Engineering
http://scientiairanica.sharif.edu

Research Note

\title{
Bayesian analysis of the Rayleigh paired comparison model under loss functions using an informative prior
}

\author{
M. Aslam ${ }^{\mathrm{a}}$ and T. Kifayat ${ }^{\mathrm{b}, *}$ \\ a. Department of Mathematics and Statistics, Riphah International University, Islamabad, Pakistan. \\ b. Department of Statistics, Quaid-i-Azam University, Islamabad, Pakistan. \\ Received 29 March 2015; received in revised form 25 November 2016; accepted 6 March 2017
}

\author{
KEYWORDS \\ Paired comparisons; \\ Rayleigh distribution; \\ Informative prior; \\ Bayes factor; \\ Loss function
}

\begin{abstract}
Considering a number of Paired Comparison (PC) models existing in the literature, the posterior distribution for the parameters of the Rayleigh PC model is derived in this paper using the informative priors: Conjugate and Dirichlet. The values of the hyperparameters are elicited using prior predictive distribution. The preferences for the data of cigarette brands, such as Goldleaf (GL), Marlboro (ML), Dunhill (DH), and Benson \& Hedges (BH), are collected based on university students' opinions. The posterior estimates of the parameters are obtained under the loss functions: Quadratic Loss Function (QLS), Weighted Loss Function (WLS), and Squared Error Loss Function (SELF) with their risks. The preference and predictive probabilities are investigated. The posterior probabilities are evaluated with respect to the hypotheses of two parameters comparison. In this respect, the graphs of marginal posterior distributions are presented, and appropriateness of the model is tested by Chi-Square.

(C) 2018 Sharif University of Technology. All rights reserved.
\end{abstract}

\section{Introduction}

In the method of $\mathrm{PC}$, items are presented in pairs to one or more judges; for each pair, a judge selects the item that best satisfies the specified judgment criterion. The importance of PC models has been illustrated through literature, as given below.

Aslam [1] proposed methods for elicitation of hyperparameters of Bradley-Terry model. Three methods for elicitation are recommended for the case of two treatments and one method for the general case. Cattelan [2] presented the extensions in the Thurstonian and Bradley-Terry models on how to account for object- and subject-specific covariates. Models

\footnotetext{
*. Corresponding author.

E-mail addresses: m.aslam@riphah.edu.pk (M. Aslam); tanveerkhan@stat.qau.edu.pk (T. Kifayat)
}

of dependent comparisons were also considered. A pairwise likelihood approach was estimated for models of dependent PC data. Moreover, a simulated study was also carried out. The Beta distribution was used as a prior for the Binomial by Chaloner and Duncan [3]. The hyperparameters were elicited by the method of PM (Posterior Mode). Gavasakar discussed two techniques for hyperparameters elicitation of the Conjugate Beta distribution for a Binomial model [4]. The results of posterior mode and imaginary-based methods are compared. Kadane and Wolfson [5] studied general versus application-specific methods and predictive versus structural techniques of elicitation. Liu and Shih [6] analyzed the PC data under decision trees. A scoring system assigned ' 2 ' to a win, ' 1 ' to a tie, and zero to a loss for each PC; total scores are counted. The GUIDE regression tree method was used for the scores as multi response, and average scores of the objects are presented on the preference scale to the objects in each terminal node. Similarly, prefer- 
ence ranking is identical to the Bradley-Terry model, considering the scoring system. The Bradley-Terry model was often used for ranking contestants in sport tournaments. Masarotto and Varin [7] proposed the method of Lasso type and categorized the contestants with the same efficiency in the same group. The advantage of the suggested method is that interpretation of ranking and prediction with respect to standard maximum likelihood is done easily. For numerical illustration, the dataset of the National Football league 2010-2011 and the American college Hockey Men's Division I 2009-2010 was used. For the consistency of PCs, Pankratova and Nedashkovskaya [8] examined the equivalence of the indicators. Furthermore, the calculation of the weights of alternatives decisions on the basis of primary and adjusted matrices of PCs leads to a variety of alternatives ranking. Further, the method of estimating the consistency of PCs was also given. Tutz and Schauberger [9] considered a general latent trait model for the assessment of sports' competitions. This model uses the consequences of playing at home, which can differ over teams. The team-specific explanatory variables are covered by the model. Further, the methods are examined by the performance and dependence on the budget for football teams of the German Bundesliga. There are many ways to rank the football teams, one of which is a double round-robin system. Another criterion is scoring higher number of goals during a competition. However, Veghes [10] proposed the method of PC approach and tested the results of the Romanian First Division. Yan et al. [11] studied the Bradley-Terry model; the PCs may be sparse and exist in some pairs. They showed that asymptotic results similar to Simons and Yao's continue to hold under a simple condition that controls sparsity. Simulation study was also carried out.

The model and notations are defined in Section 2. Section 3 presents the posterior distributions used for Bayesian analysis. Elicitation of the hyperparameters and the Rayleigh PC model under Bayesian context is analyzed in Section 4. Conclusion of the study is reviewed in Section 5 .

\section{Model and notations}

The Rayleigh PC model and its notation are expressed in this section. Let $r_{i j}$ be a random variable associated with the rank of the treatments in the $k$ th repetition of treatment pair $\left(T_{i}, T_{j}\right)$, where $i \neq j ; i \geq 1, j \leq m$; $k=1,2, \ldots, n_{i j}$; and $m$ is the number of observations.

- $r_{i . i j k}=1$ or 0 accordingly, as treatment $T_{i}$ is preferred to treatment $T_{j}$ or not in the $k$ th repetition of comparison;

- $r_{j . i j k}=1$ or 0 accordingly, as treatment $T_{j}$ is preferred to treatment $T_{i}$ or not in the $k$ th repetition of comparison;

- $r_{i . i j}=\sum_{k} r_{i . i j k}=$ the number of times treatment $T_{i}$ is preferred to treatment $T_{j}$;

- $r_{j . i j}=\sum_{k} r_{j . i j k}=$ the number of times treatment $T_{j}$ is preferred to treatment $T_{i}$;

- $n_{i j}=$ the number of times treatment $T_{i}$ is compared with treatment $T_{j}$,

- $n_{i j}=r_{i . i j}+r_{j . i j}$.

As the Rayleigh distribution can be used in communication theory, in a paired comparison, perception of the preference for one object is communicated to the other object in a pair; for this reason, the Rayleigh distribution may be considered for PC model. Model criterion proposed by Stern [12] was used to develop the Rayleigh PC model. The probability of the preference of $T_{i}$ over $T_{j}$ is denoted by $\eta_{i . i j}$ and defined as follows:

$$
\begin{aligned}
& \eta_{i . i j}=P\left(T_{i} \geq T_{j}\right), \\
& \eta_{i . i j}=\int_{0}^{\infty} \int_{t_{j}}^{\infty} \frac{t_{i}}{\beta_{i}^{2}} e^{-\frac{t_{i}^{2}}{2 \beta_{i}^{2}}} \frac{t_{j}}{\beta_{j}^{2}} e^{-\frac{t_{j}^{2}}{2 \beta_{j}^{2}}} d t_{i} d t_{j}, \\
& \eta_{i . i j}=\frac{\beta_{i}^{2}}{\beta_{i}^{2}+\beta_{j}^{2}},
\end{aligned}
$$

where $\eta_{j . i j}$ is the probability of $T_{j}$ being preferred over $T_{i}$ which is obtained as follows:

$$
\begin{aligned}
& \eta_{j . i j}=1-\eta_{i . i j}, \\
& \eta_{j . i j}=\frac{\beta_{j}^{2}}{\beta_{i}^{2}+\beta_{j}^{2}},
\end{aligned}
$$

where $\beta_{i} ;(i<j)=1,2, \ldots, m$ are the treatment parameters. Eqs. (1) and (2) represent the model called the Rayleigh model for PC.

\section{The posterior distribution}

The posterior distribution is constituted through the combination of the prior and sample information. The posterior distribution reflects the updated beliefs from which all decisions and inferences are made:

$$
p(\beta \mid \mathbf{r}) \propto \text { prior } \times \text { likelihood. }
$$

The likelihood function of the Rayleigh PC model for the observed outcomes of trials $\mathbf{r}$ and parameters $\boldsymbol{\beta}=$ $\beta_{1}, \beta_{2}, \ldots, \beta_{4}$ is:

$$
\begin{aligned}
& l(\mathbf{r}, \boldsymbol{\beta})=\prod_{i<j=1}^{m} \frac{n_{i j} !}{r_{i j} !\left(n_{i j}-r_{i j}\right) !} \frac{\beta_{i}^{2 r_{i . i j}} \beta_{j}^{2 r_{j . i j}}}{\left(\beta_{i}^{2}+\beta_{j}^{2}\right)^{r_{i . i j}+r_{j . i j}}} \\
& \beta_{i}>0 .
\end{aligned}
$$

The constraint is imposed upon the treatment parameters $\sum_{i=1}^{m} \beta_{i}=1$; therefore, the parameters are well defined. 


\subsection{The posterior distribution using the conjugate prior}

According to Raiffa and Schlaifer [13], a distribution is said to be natural for the Conjugate in a given sampling process if its probability density function is proportional to a likelihood function corresponding to some conceivable samples from the process. The Conjugate prior of the Rayleigh PC model is as follows:

$$
p_{c}(\boldsymbol{\beta})=\prod_{i<j=1}^{m} \frac{\beta_{i}^{2 c_{i}}}{\left(\beta_{i}^{2}+\beta_{j}^{2}\right)^{c_{m+1}}}, \quad \beta>0, \quad \sum_{i=1}^{m} \beta_{i}=1
$$

where $c_{i}(i=1, \ldots, m+1)$ are the hyperparameters. The joint posterior distribution of the Rayleigh PC model parameters $\beta_{1}, \ldots, \beta_{m}$ given data, using Eq. (3) and $p(\boldsymbol{\beta})$, (prior distribution) is:

$$
\begin{aligned}
& p\left(\beta_{1}, \ldots, \beta_{m} \mid \mathbf{r}\right)=\frac{1}{K} p(\boldsymbol{\beta}) \prod_{i<j=1}^{m} \frac{n_{i j} !}{r_{i j} !\left(n_{i j}-r_{i j}\right) !} \\
& \quad \times \frac{\beta_{i}^{2 r_{i . i j}} \beta_{j}^{2 r_{j . i j}}}{\left(\beta_{i}^{2}+\beta_{j}^{2}\right)^{r_{i . i j}+r_{j . i j}}}, \\
& \beta_{i}>0, \quad \sum_{i=1}^{m} \beta_{i}=1,
\end{aligned}
$$

where $K$ is the normalizing constant, defined as:

$$
\begin{aligned}
K= & \int_{0}^{1} \int_{0}^{1-\beta_{1}} \ldots \int_{0}^{1-\beta_{1} \ldots-\beta_{m-2}} p(\boldsymbol{\beta}) \\
& \prod_{i<j=1}^{m} \frac{n_{i j} !}{r_{i j} !\left(n_{i j}-r_{i j}\right) !} \\
& \times \frac{\beta_{i}^{2 r_{i . i j}} \beta_{j}^{2 r_{j . i j}}}{\left(\beta_{i}^{2}+\beta_{j}^{2}\right)^{r_{i . i j}+r_{j . i j}}} d \beta_{m-1} \ldots d \beta_{2} d \beta_{1} .
\end{aligned}
$$

The marginal posterior distribution of the Rayleigh PC model parameter of $\beta_{1}$ given data, under Conjugate prior using Eqs. (3) and (4), is:

$$
\begin{aligned}
& p\left(\beta_{1} \mid \mathbf{r}\right)=\int_{0}^{1-\beta_{1}} \int_{0}^{1-\beta_{1} \ldots-\beta_{m-2}} p_{c}(\boldsymbol{\beta}) \\
& \prod_{i<j=1}^{m} \frac{n_{i j} !}{r_{i j} !\left(n_{i j}-r_{i j}\right) !} \\
& \quad \times \frac{\beta_{i}^{2 r_{i . i j}} \beta_{j}^{2 r_{j . i j}}}{\left(\beta_{i}^{2}+\beta_{j}^{2}\right)^{r_{i . i j}+r_{j . i j}}} d \beta_{m-1} \ldots d \beta_{2}, \\
& \beta_{i}>0, \quad \sum_{i=1}^{m} \beta_{i}=1 .
\end{aligned}
$$

\subsection{The posterior distribution using the Dirichlet prior}

The Dirichlet distribution is used as another informative prior, which is compatible with the parameters of
Table 1. Data of cigarette brands.

\begin{tabular}{cccc}
\hline Cigarette brands & $r_{\boldsymbol{i} . \boldsymbol{j} \boldsymbol{j}}$ & $r_{\boldsymbol{j} \boldsymbol{i j}}$ & $n_{\boldsymbol{i j}}$ \\
\hline$(\mathrm{GL}, \mathrm{ML})$ & 12 & 8 & 20 \\
$(\mathrm{GL}, \mathrm{DH})$ & 13 & 7 & 20 \\
$(\mathrm{GL}, \mathrm{BH})$ & 10 & 10 & 20 \\
$(\mathrm{ML}, \mathrm{DH})$ & 14 & 6 & 20 \\
$(\mathrm{ML}, \mathrm{BH})$ & 7 & 13 & 20 \\
$(\mathrm{DH}, \mathrm{BH})$ & 9 & 11 & 20 \\
\hline
\end{tabular}

the Rayleigh PC model as follows:

$$
\begin{aligned}
& p_{d}(\boldsymbol{\beta})=\frac{\Gamma\left(d_{1}+\ldots+d_{m}\right)}{\Gamma\left(d_{1}\right) \ldots \Gamma\left(d_{m}\right)} \prod_{i=1}^{m} \beta_{i}^{d_{i}-1}, \\
& \beta_{i}>0, \quad \sum_{i=1}^{m} \beta_{i}=1,
\end{aligned}
$$

where $d_{i}(i=1, \ldots, m)$ are the hyperparameters. The marginal posterior distribution of the Rayleigh PC model parameter of $\beta_{1}$ given data under the Dirichlet prior using Eqs. (3) and (6) is:

$$
\begin{aligned}
& p\left(\beta_{1} \mid \mathbf{r}\right)=\int_{0}^{1-\beta_{1}} \int_{0}^{1-\beta_{1} \ldots-\beta m-2} p_{d}(\boldsymbol{\beta}) \\
& \prod_{i<j=1}^{m} \frac{n_{i j} !}{r_{i j} !\left(n_{i j}-r_{i j}\right) !} \\
& \quad \times \frac{\beta_{i}^{2 r_{i . i j}} \beta_{j}^{2 r_{j . i j}}}{\left(\beta_{i}^{2}+\beta_{j}^{2}\right)^{r_{i . i j}+r_{j . i j}}} d \beta_{m-1} \ldots d \beta_{2}, \\
& \beta_{i}>0, \sum_{i=1}^{m} \beta_{i}=1 .
\end{aligned}
$$

The dataset of 20 observations of four cigarette brands commonly used among the students of Quaidi-Azam University is presented in Table 1.

\section{Elicitation}

Elicitation is the exercise of excavating the probabilities and utilities from individuals regarding uncertain events or phenomena. There are two main modules to this exercise: First, the psychological background on how individuals can best answer questions for probability encoding; second, the statistical aspects of how to use the answers to determine a prior distribution. Aslam [1] focused on the procedure of elicitation using prior predictive distribution. Three different methods are defined to elicit the hyperparameters: prior predictive probabilities, predictive mode, and a confidence level and elicitation of confidence levels.

The elicitation method of confidence levels through prior predictive distribution for the hyperparameters of the prior density for the parameter of the 
model suggested in [1] is used in the paper to elicit the hyperparameters.

The confidence levels of the prior predictive distribution would be elicited for specific intervals. The hyperparameters can be elicited through the following function:

$$
\Psi(\underline{c})=\min (\underline{c}) \sum_{i=1}^{m}\left|(\mathrm{CCL})_{h}-(\mathrm{ECL})_{h}\right| .
$$

As ' $m$ ' is the number of interval considered for elicitation, $\underline{c}$ is a vector of elicited hyperparameters, CCL is the confidence level of hyperparameters, and ECL is the elicited confidence level. The set of hyperparameters with minimum value of $\Psi(\underline{c})$ in the above equation is considered as the elicited values of hyperparameters.

\subsection{Elicitation of hyperparameters of the conjugate prior}

The prior predictive distribution of using the Conjugate prior is:

$$
\begin{aligned}
p\left(r_{i j}\right) & =\int_{\beta} \prod_{i<j=1}^{m=4} \frac{n_{i j} !}{r_{i j} !\left(n_{i j}-r_{i j}\right) !} \\
& \times \frac{\beta_{i}^{2\left(r_{i . i j}+c_{i}\right)}\left(1-\beta_{i}\right)^{2\left(r_{j . i j}+c_{j}\right)}}{\left(\beta_{i}^{2}+\left(1-\beta_{i}\right)^{2}\right)^{\left(n_{i j}+c_{5}\right)}} d \beta_{i} .
\end{aligned}
$$

Herein, $c_{1}, c_{2}, c_{3}, c_{4}$, and $c_{5}$ are the hyperparameters. The six expert confidence levels at different intervals, using the Conjugate prior predictive distribution, are as follows:

$$
\begin{aligned}
& \sum_{r_{12}=0}^{5} p\left(r_{12}\right)=0.05, \quad \sum_{r_{13}=1}^{6} p\left(r_{13}\right)=0.02, \\
& \sum_{r_{14}=2}^{7} p\left(r_{14}\right)=0.04, \quad \sum_{r_{23}=0}^{6} p\left(r_{23}\right)=0.03, \\
& \sum_{r_{24}=3}^{8} p\left(r_{24}\right)=0.08, \quad \sum_{r_{34}=2}^{7} p\left(r_{34}\right)=0.05 .
\end{aligned}
$$

The program was designed in the SAS package for the elicitation of hyperparameters using the Conjugate prior. The elicited hyperparameters are given in Table 2 .

\subsection{Elicitation of hyperparameters of the Dirichlet prior}

The prior predictive distribution of using the Dirichlet prior is:

$$
\begin{gathered}
p\left(r_{i j}\right)=\int_{\beta} \prod_{i<j=1}^{m=4} \frac{n_{i j} !}{r_{i j} !\left(n_{i j}-r_{i j}\right) !} \times \frac{\Gamma\left(d_{i}+d_{j}\right)}{\Gamma d_{i} \Gamma d_{j}} \\
\frac{\beta_{i}^{2 r_{i . i j}+d_{i}-1}\left(1-\beta_{i}\right)^{2 r_{j . i j}+d_{j}-1}}{\left(\beta_{i}^{2}+\left(1-\beta_{i}\right)^{2}\right)^{n_{i j}}} d \beta_{i} .
\end{gathered}
$$

Table 2. Elicited hyperparameters of the conjugate prior.

\begin{tabular}{ccccc}
\hline $\boldsymbol{c}_{\boldsymbol{1}}$ & $\boldsymbol{c}_{\boldsymbol{2}}$ & $\boldsymbol{c}_{\boldsymbol{3}}$ & $\boldsymbol{c}_{\boldsymbol{4}}$ & $\boldsymbol{c}_{\boldsymbol{5}}$ \\
\hline 2.27 & 2.25 & 2.43 & 2.06 & 4.27 \\
\hline
\end{tabular}

Table 3. Elicited hyperparameters of the Dirichlet prior

\begin{tabular}{cccc}
\hline $\boldsymbol{d}_{\mathbf{1}}$ & $\boldsymbol{d}_{\boldsymbol{2}}$ & $\boldsymbol{d}_{\boldsymbol{3}}$ & $\boldsymbol{d}_{\boldsymbol{4}}$ \\
\hline 2.11 & 1.76 & 1.84 & 1.86 \\
\hline
\end{tabular}

Table 4. The posterior estimate.

\begin{tabular}{ccc}
\hline \multirow{2}{*}{ Parameters } & \multicolumn{2}{c}{ Posterior estimate } \\
\cline { 2 - 3 } & Conjugate-prior & Dirichlet-prior \\
\hline$\beta_{1}$ & 0.27981 & 0.28245 \\
$\beta_{2}$ & 0.24226 & 0.24139 \\
$\beta_{3}$ & 0.20556 & 0.20174 \\
$\beta_{4}$ & 0.27238 & 0.27443 \\
\hline
\end{tabular}

Herein, $d_{1}, d_{2}, d_{3}$, and $d_{4}$ are hyperparameters.

The six expert confidence levels at different intervals, using the Dirichlet prior predictive distribution, are:

$$
\begin{aligned}
& \sum_{r_{12}=2}^{5} p\left(r_{12}\right)=0.05, \quad \sum_{r_{13}=2}^{4} p\left(r_{13}\right)=0.03, \\
& \sum_{r_{14}=2}^{4} p\left(r_{14}\right)=0.05, \quad \sum_{r_{23}=2}^{4} p\left(r_{23}\right)=0.02, \\
& \sum_{r_{24}=3}^{4} p\left(r_{24}\right)=0.08, \quad \sum_{r_{34}=3}^{6} p\left(r_{34}\right)=0.06 .
\end{aligned}
$$

The program was designed in the SAS package for the elicitation of hyperparameters. Table 3 comprises the elicited hyperparameters of the Dirichlet prior, and these values are used for further analysis.

\section{The Rayleigh PC model under Bayesian analysis}

In this section, the Rayleigh PC model is studied under Bayesian analysis using the posterior estimates of parameter. The preference probabilities are also calculated. The predictive probabilities for the single future values of the parameters are evaluated. The posterior probabilities are obtained. The appropriateness of the model is also investigated.

\subsection{The posterior estimate}

The posterior means are computed as the estimates of parameter. The posterior estimates (mean) of the Rayleigh PC model using the Conjugate and Dirichlet priors are calculated and given in Table 4 . 


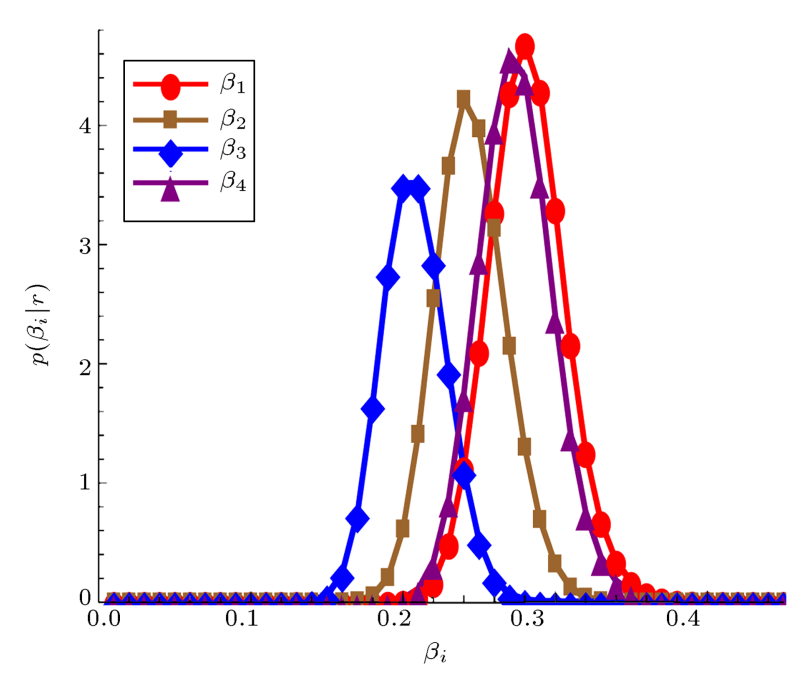

Figure 1. The marginal posterior distributions for $\beta_{i}$ of the Rayleigh model using conjugate prior.

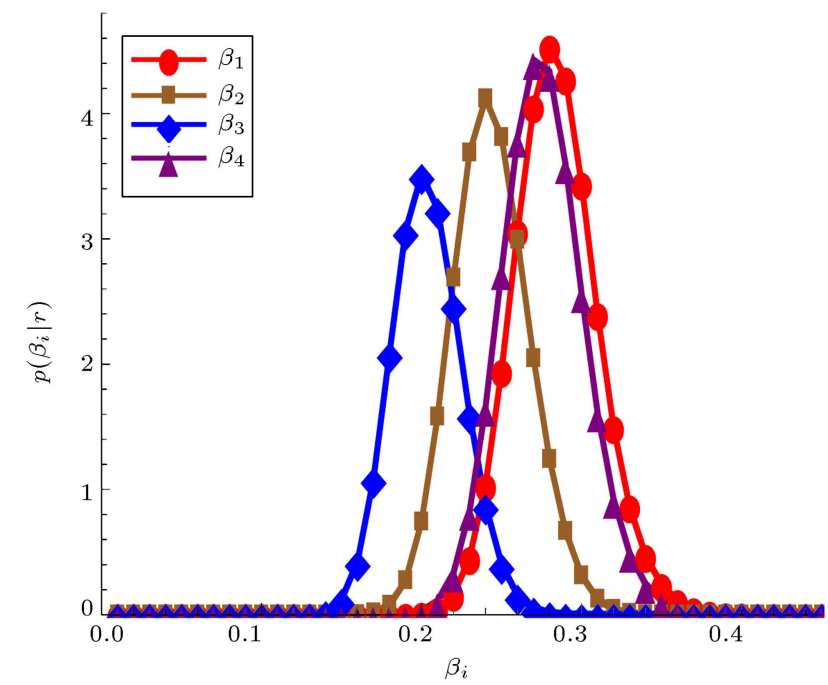

Figure 2. The marginal posterior distributions for $\beta_{i}$ of the Rayleigh model using Dirichlet prior.

As noticed from Table 4, the GL is the highest favored cigarette brand among the students as the posterior estimates are the largest. The $\mathrm{BH}$ is favored more than the ML and $\mathrm{DH}$. Moreover, the $\mathrm{DH}$ is the least favorable brand among the students as the parameter estimates are the smallest.

\subsection{Graphs of the marginal posterior distribution}

The graphs of the marginal posterior distribution for the Rayleigh PC model using dataset in Table 1 for the Conjugate and Dirichlet priors are drawn. Figures 1 and 2 have a symmetrical shape.

\subsection{The Bayes estimator under loss functions} This section contains the derivation of the Bayes estimator under loss functions. The Bayes decision is a decision ' $\beta$ ' which minimizes risk function and $\beta^{*}$ is the best decision. If the decision is the choice of an estimator, then the Bayes decision is a Bayes estimator. We use three squared loss functions.

\subsubsection{Quadratic loss function}

The quadratic loss function is given as:

$$
L_{1}\left(\beta, \beta^{*}\right)=\left(1-\frac{\beta^{*}}{\beta}\right)^{2},
$$

$\beta^{*}=\frac{E\left(\beta^{-1}\right)}{E\left(\beta^{-2}\right)}$, is the Bayes estimator, and $\rho_{1}\left(\beta^{*}\right)=$ $1-\frac{E\left(\beta^{-1}\right)^{2}}{E\left(\beta^{-2}\right)}$, is the Bayes posterior risk.

\subsubsection{Weighted loss function}

The weighted loss function is given as follows:

$$
L_{2}\left(\beta, \beta^{*}\right)=\frac{\beta-\beta^{*}}{\beta},
$$

$\beta^{*}=\frac{1}{E\left(\beta^{-1}\right)}$, is the Bayes estimator, and $\rho_{2}\left(\beta^{*}\right)=$ $E(\beta)-\frac{1}{E\left(\beta^{-1}\right)}$, is the Bayes posterior risk.

\subsubsection{Squared error loss function}

The squared loss function is given as:

$$
L_{3}\left(\beta, \beta^{*}\right)=\left(\beta-\beta^{*}\right)^{2}
$$

$\beta^{*}=E(\beta)$, is the Bayes estimator, and $\rho_{2}\left(\beta^{*}\right)=$ $E\left(\beta^{2}\right)-E(\beta)^{2}$, is the Bayes posterior risk.

Table 5 comprises the Bayes estimators and posterior risk under loss functions. The Bayes posterior risks are given in parentheses.

Table 5. The Bayes estimator under loss functions.

\begin{tabular}{cccc}
\hline \multirow{2}{*}{ Parameters } & \multicolumn{3}{c}{ Conjugate-prior } \\
\cline { 2 - 4 } & $\boldsymbol{L}_{\mathbf{1}}$ & $\boldsymbol{L}_{\mathbf{2}}$ & $\boldsymbol{L}_{\mathbf{3}}$ \\
\hline \multirow{2}{*}{$\beta_{1}$} & 0.27506 & 0.27743 & 0.27981 \\
$\beta_{2}$ & $(0.00855)$ & $(0.00238)$ & $(0.00067)$ \\
& 0.23781 & 0.24003 & 0.24226 \\
$\beta_{3}$ & $(0.00927)$ & $(0.00222)$ & $(0.00054)$ \\
& 0.20125 & 0.20341 & 0.20556 \\
$\beta_{4}$ & $(0.01061)$ & $(0.00215)$ & $(0.00044)$ \\
& 0.26770 & 0.27003 & 0.27238 \\
Parameters & $(0.00866)$ & $(0.00234)$ & $(0.00064)$ \\
\cline { 2 - 4 }$\beta_{1}$ & \multicolumn{3}{c}{ Dirichlet-prior } \\
\hline \multirow{2}{*}{$\beta_{2}$} & 0.27741 & 0.27993 & 0.28245 \\
& $(0.00898)$ & $(0.00252)$ & $(0.000071)$ \\
$\beta_{3}$ & 0.23663 & 0.23901 & 0.24139 \\
& $(0.00996)$ & $(0.00238)$ & $(0.00057)$ \\
$\beta_{4}$ & 0.19708 & 0.19942 & 0.20174 \\
& $(0.01171)$ & $(0.00232)$ & $(0.00047)$ \\
& 0.26946 & 0.27194 & 0.27443 \\
& $(0.00913)$ & $(0.00249)$ & $(0.00068)$ \\
\hline
\end{tabular}


The Bayes estimators under loss function, $L_{3}$, have overall minimum risk, more than those under $L_{1}$ and $L_{2}$ using both the Conjugate and Dirichlet priors. These estimates are used to find the preference probabilities.

\subsection{The preference probabilities}

To check out the supremacy among the cigarette brands, the preference probabilities are computed in Table 6 . The preference probabilities signify that the GL is considered to be a greatly preferred cigarette brand, where the $\mathrm{BH}$ is more preferred than the ML. The $\mathrm{DH}$ is given the lowest preference among the brands.

\subsection{The predictive probabilities}

The single future preference of a treatment over another treatment is forecast by the predictive probabilities. It is concluded from Table 7 that there is a $56.43 \%$ chance that GL will be preferred to ML and a $64.94 \%$ chance that GL will be preferred to $\mathrm{DH}$ in a single future PC. Similarly, there is a $36.52 \%$ chance that DH will be preferred to $\mathrm{BH}$ in a single future $\mathrm{PC}$.

\subsection{The hypotheses testing}

The hypotheses testing define the evidence of the quality for one model specification over another. In Bayesian analysis, the posterior probabilities are directly calculated, and one may decide between hy-

Table 6. The preference probabilities.

\begin{tabular}{cccc}
\hline Pairs & $\phi_{i . i j}$ & $\begin{array}{c}\text { Conjugate- } \\
\text { prior }\end{array}$ & $\begin{array}{c}\text { Dirichlet- } \\
\text { prior }\end{array}$ \\
\hline$(\mathrm{GL}, \mathrm{ML})$ & $\phi_{1.12}$ & 0.57156 & 0.57790 \\
$(\mathrm{GL}, \mathrm{DH})$ & $\phi_{1.13}$ & 0.64948 & 0.66218 \\
$(\mathrm{GL}, \mathrm{BH})$ & $\phi_{1.14}$ & 0.51345 & 0.51439 \\
$(\mathrm{ML}, \mathrm{DH})$ & $\phi_{2.23}$ & 0.58141 & 0.58877 \\
$(\mathrm{ML}, \mathrm{BH})$ & $\phi_{2.24}$ & 0.44167 & 0.43621 \\
$(\mathrm{DH}, \mathrm{BH})$ & $\phi_{3.34}$ & 0.36287 & 0.35082 \\
\hline
\end{tabular}

Table 7. The predictive probabilities.

\begin{tabular}{cccc}
\hline Pairs & $\boldsymbol{P}_{\boldsymbol{i j}}$ & $\begin{array}{c}\text { Conjugate- } \\
\text { prior }\end{array}$ & $\begin{array}{c}\text { Dirichlet- } \\
\text { prior }\end{array}$ \\
\hline (GL, ML) & $P_{12}$ & 0.56427 & 0.57120 \\
$(\mathrm{GL}, \mathrm{DH})$ & $P_{13}$ & 0.64944 & 0.65744 \\
$(\mathrm{GL}, \mathrm{BH})$ & $P_{14}$ & 0.51598 & 0.51661 \\
$(\mathrm{ML}, \mathrm{DH})$ & $P_{23}$ & 0.58835 & 0.58988 \\
$(\mathrm{ML}, \mathrm{BH})$ & $P_{24}$ & 0.45151 & 0.44516 \\
$(\mathrm{DH}, \mathrm{BH})$ & $P_{34}$ & 0.36521 & 0.35770 \\
\hline
\end{tabular}

Table 8. The posterior probabilities.

\begin{tabular}{ccccc}
\hline \multirow{2}{*}{ Hypotheses } & \multicolumn{2}{c}{ Conjugate-prior } & \multicolumn{2}{c}{ Dirichlet-prior } \\
\cline { 2 - 5 } & $\boldsymbol{p}_{\boldsymbol{i} \boldsymbol{j}}$ & $\boldsymbol{B}$ & $\boldsymbol{p}_{\boldsymbol{i} \boldsymbol{j}}$ & $\boldsymbol{B}$ \\
\hline$\beta_{1} \geq \beta_{2}$ & 0.59442 & 1.46560 & 0.62618 & 1.67508 \\
$\beta_{1} \geq \beta_{3}$ & 0.91732 & 11.09482 & 0.93796 & 15.11863 \\
$\beta_{1} \geq \beta_{4}$ & 0.47263 & 0.89620 & 0.46819 & 0.88037 \\
$\beta_{2} \geq \beta_{3}$ & 0.77775 & 3.49944 & 0.76825 & 3.31499 \\
$\beta_{2} \geq \beta_{4}$ & 0.05565 & 0.05893 & 0.05352 & 0.05654 \\
$\beta_{3} \geq \beta_{4}$ & 0.00272 & 0.00273 & 0.00243 & 0.00244 \\
\hline
\end{tabular}

potheses. The hypotheses can be defined as follows:

$$
H_{i j}: \beta_{i} \geq \beta_{j} \text { vs. } H_{j i}: \beta_{i}<\beta_{j}
$$

The posterior probability for hypothesis $H_{i j}$ is:

$$
p_{i j}=\int_{\nu=0}^{1} \int_{\delta=\nu}^{(1+\nu) / 2} p(\nu, \delta \mid \mathbf{r}) d \delta d \nu .
$$

The posterior probability for hypothesis $H_{j i}$ is:

$$
q_{i j}=1-p_{i j}
$$

where $\delta=\beta_{i}$ and $\nu=\beta_{i}-\beta_{j}$.

The Bayes factor is used as the decision rule for the hypotheses. It can be interpreted as the odds for $H_{i j}$ to $H_{j i}$ that are given by the data. Jeffreys [14] presented the following typology by comparing $H_{i j}$ to $H_{j i}$ :

$$
\begin{aligned}
& B \geq 1 \quad \text { supports } H_{i j} \\
& 10^{-0.5} \leq B \leq 1 \text { minimal evidence against } H_{i j} \\
& 10^{-1} \leq B \leq 10^{-0.5} \text { substantial evidence against } H_{i j} \\
& 10^{-2} \leq B \leq 10^{-1} \text { strong evidence against } H_{i j} \\
& B \leq 10^{-2} \quad \text { decisive evidence against } H_{i j}
\end{aligned}
$$

From Table 8 , it is observed that $H_{12}, H_{13}$, and $H_{23}$ are supported, where $H_{14}$ has minimal evidence against $H_{41}$. It is concluded that $H_{24}$ has strong evidence against $H_{42}$ and $H_{34}$ has decisive evidence against the alternative hypothesis.

\subsection{Appropriateness of the model}

The Chi-square test is used for the appropriateness of the models. The hypothesis is defined as: 
Table 9. Appropriateness of the Rayleigh PC model.

\begin{tabular}{ccc}
\hline & \multicolumn{2}{c}{ Rayleigh PC model } \\
\cline { 2 - 3 } & Conjugate-prior & Dirichlet-prior \\
\hline$\chi^{2}$ & 2.57469 & 2.56015 \\
\hline$P-$ value & 0.5381 & 0.5355 \\
\hline & Bradley Terry & PC Model \\
\cline { 2 - 3 } & Conjugate-Prior & Dirichlet-Prior \\
\hline$\chi^{2}$ & 2.57071 & 2.55719 \\
\hline$P-$ value & 0.53735 & 0.53495 \\
\hline
\end{tabular}

$H_{0}$ : The model is true for some values of $\boldsymbol{\beta}=\beta_{0}$,

$H_{1}$ : $\quad$ The model is not true for any values of the parameters,

where $\boldsymbol{\beta}=\beta_{1}, \beta_{2}, \ldots, \beta_{m}$ is the vector of the unknown parameters, $\beta_{i}>0 . \chi^{2}$ has the following form:

$$
\chi^{2}=\sum_{i<j}^{m}\left\{\frac{\left(r_{i . i j}-\hat{r}_{i . i j}\right)^{2}}{\hat{r}_{j . i j}}+\frac{\left(r_{j . i j}-\hat{r}_{j . i j}\right)^{2}}{\hat{r}_{j . i j}}\right\},
$$

with $(m-1)(m-2) / 2$ degrees of freedom [15].

The expected number of preferences is obtained by the following form:

$$
\hat{r}_{i . i j}=r_{i j} \frac{\beta_{i}^{2}}{\phi_{i j}} \text { and } \hat{r}_{j . i j}=r_{i j} \frac{\beta_{j}^{2}}{\phi_{i j}},
$$

where $\phi_{i j}=\beta_{i}^{2}+\beta_{j}^{2} . \quad r_{i j}$ and $r_{j . i j}$ are the observed numbers of preferences from the dataset given in Table 1. The appropriateness of the Rayleigh PC model is compared with the Bradley Terry PC model in Table 9.

From Table 9, it is interpreted that $\chi^{2}$ has high $P$-values. Therefore, the both models are good fit.

\section{Conclusion}

A study was conducted with respect to the developed Rayleigh PC model in this study. The model was analyzed under Bayesian paradigm using the Conjugate and Dirichlet priors. The prior predictive distribution for elicitation of hyperparameters was used. The analysis of the study is based on the dataset of four cigarette brands: Goldleaf, Marlboro, Dunhill, and Benson \& Hedges collected from Quaid-i-Azam University, Islamabad, Pakistan. The loss functions include Quadratic Loss Function (QLS), Weighted Loss Function (WLS), and Squared Error Loss Function (SELF) for the estimation of parameters. The Bayes estimators under loss function SELF have the overall minimum risk, as compared to QLS and WLS, for both the Conjugate and Dirichlet priors. The posterior estimates were obtained. The predictive and preference probabilities were estimated. The appropriateness of the model was calculated. On the basis of the estimates, it is concluded that Goldleaf is the most preferred cigarette among students, while Dunhill is the least preferred cigarette brand.

\section{References}

1. Aslam, M. "An application of prior predictive distribution to elicit the prior density", Journal of Statistical Theory and Applications, 2(1), pp. 70-83 (2003).

2. Cattelan, M. "Models for paired comparison data: A review with emphasis on dependent data", Statistical Science, 27(3), p. 412433 (2012).

3. Chaloner, K.M. and Duncan, G.T. "Assessment of a beta prior distribution: PM elicitation", The Statistician, 32(1/2), pp. 174-180 (1983).

4. Gavasakar, U. "A comparison of two elicitation methods for a prior distribution for a binomial parameter", Management Science, 34(6), pp. 784-790 (1998).

5. Kadane, J.B. and Wolfson, L.J. "Experiences in elicitation", The Statistician, 47(1), pp. 3-19 (1998).

6. Liu, K.H. and Shih, Y.S. "Score-scale decision tree for paired comparison data", Statistica Sinica, 26, pp. 429-444 (2016).

7. Masarotto, G. and Varin, C. "The ranking Lasso and its application to sport tournaments", The Annals of Applied Statistics, 6(4), pp. 1949-1970 (2012).

8. Pankratova, N. and Nedashkovskaya, N. "The method of estimating the consistency of paired comparisons", International Journal Information Technologies \& Knowledge, 7(4), pp. 347-361 (2013).

9. Tutz, G. and Schauberger, G. "Extended ordered paired comparison models with application to football data from German Bundesliga", AStA Advances in Statistical Analysis, 99(2), pp. 209-227 (2015).

10. Veghes, C. "Using the paired comparison method in improving soccer rankings: The case of the Romanian first division", Annales Universitatis Apulensis Series Oeconomica, 16(2), pp. 379-388 (2014).

11. Yan, T., Yang, Y., and Xu, J. "Sparse paired comparisons in the Bradley-Terry model", Statistica Sinica, 22, pp. 1305-1318 (2012).

12. Stern, H. "A continuum of paired comparisons models", Biometrika, $\mathbf{7 7}(2)$, pp. 265-273 (1990).

13. Raiffa, H. and Schlaifer, R., Applied Statistical Decision Theory, Division of Research, Harvard Business School, Boston (1961). 
14. Jeffreys, H., Theory of Probability, Oxford University Press, Oxford UK (1961).

15. Abbas, N. and Aslam, M. "Prioritizing the items through paired comparison models, a Bayesian approach", Pakistan Journal of Statistics, 25(1), pp. 5969 (2009).

\section{Biographies}

Muhammad Aslam is a Professor of Statistics at Riphah International University, Islamabad, Pakistan and the former Chairperson at the Statistics Depart- ment, Quaid-i-Azam University, Islamabad, Pakistan. He has done his $\mathrm{PhD}$ in the Statistics from University of Wales. He has published more than 100 publications. His research interests include Bayesian inference, reliability analysis, and mixture distributions.

Tanveer Kifayat did her MPhil degree in the Statistics from the Quaid-i-Azam University, Islamabad, Pakistan. She has two publications. Currently she is a PhD scholar at the Statistics Department, Quaid-iAzam University, Islamabad, Pakistan. 